\title{
Diabetes, biochemical markers of bone turnover, diabetes control, and bone
}

\author{
Jakob Starup-Linde ${ }^{1,2} *$ \\ ${ }^{1}$ Faculty of Health, Aalborg University, Aalborg, Denmark \\ 2 Department of Endocrinology and Internal Medicine, Aarhus University Hospital THG, Aarhus, Denmark
}

\section{Edited by:}

Peter Vestergaard, Aarhus University

Hospital, Denmark

\section{Reviewed by:}

David M. Findlay, University of Adelaide, Australia

Julie Clare Crockett, University of

Aberdeen, UK

\section{*Correspondence}

Jakob Starup-Linde, Department of Endocrinology and Metabolism, Aarhus University Hospital THG, Tage Hansens Gade 2, DK-8000 Aarhus, Denmark.

e-mail: jakolind@rm.dk
Diabetes mellitus is known to have late complications including micro vascular and macro vascular disease. This review focuses on another possible area of complication regarding diabetes; bone. Diabetes may affect bone via bone structure, bone density, and biochemical markers of bone turnover. The aim of the present review is to examine in vivo from humans on biochemical markers of bone turnover in diabetics compared to non-diabetics. Furthermore, the effect of glycemic control on bone markers and the similarities and differences of type 1- and type 2-diabetics regarding bone markers will be evaluated. A systematic literature search was conducted using PubMed, Embase, Cinahl, and SveMed+ with the search terms: "Diabetes mellitus," "Diabetes mellitus type 1," "Insulin dependent diabetes mellitus," "Diabetes mellitus type 2," "Non-insulin dependent diabetes mellitus," "Bone," "Bone and Bones," "Bone diseases," "Bone turnover," "Hemoglobin A Glycosylated," and "HbA1C." After removing duplicates from this search 1,188 records were screened by title and abstract and 75 records were assessed by full text for inclusion in the review. In the end 43 records were chosen. Bone formation and resorption markers are investigated as well as bone regulating systems. T1D is found to have lower osteocalcin and CTX, while osteocalcin and tartrate-resistant acid are found to be lower in T2D, and sclerostin is increased and collagen turnover markers altered. Other bone turnover markers do not seem to be altered in T1D or T2D. A major problem is the lack of histomorphometric studies in humans linking changes in turnover markers to actual changes in bone turnover and further research is needed to strengthen this link.

Keywords: diabetes mellitus, bone, bone turnover, markers of bone turnover, biochemical markers, glycemic control

\section{INTRODUCTION}

Diabetes mellitus is a common disease in most parts of the world (WHO, 2012; Wikipedia, 2012). Well known late complications of diabetes are micro vascular disease including nephropathy, retinopathy, neuropathy, and macro vascular disease such as acute coronary syndrome, claudicatio intermittens, and stroke (American Diabetes Association, 2012). However, the bone turnover and thus the skeletal integrity may also be affected by diabetes, and diabetic bone disease can represent a hitherto overlooked complication of diabetes. A meta-analysis reported lower bone mineral density (BMD) $z$-score in T1D, but higher $z$-score in

Abbreviations: 1,25OHD, 1,25 dihydroxy vitamin D; 25OHD, 25 hydroxy vitamin $\mathrm{D}$; AP, alkaline phosphatase; BAP, bone-specific alkaline phosphatase; BMD, bone mass density; BMI, body mass index; CICP, collagen type $1 \mathrm{C}$ propeptide; CTX, Cterminal cross-linked telopeptide of type-I collagen; DPD, deoxypyridinoline; FPG, fasting plasma glucose; HbAlC, glycated hemoglobin; HP, hydroxyproline; IDD, insulin dependent diabetes; IGF-1, insulin like growth factor-1; NIDD, non-insulin dependent diabetes; NTX, N-terminal cross-linked telopeptide of type-I collagen; OC, osteocalcin; OPG, osteoprotegerin; $\mathrm{P} 1 \mathrm{CP}$, procollagen type 1 carboxyl terminal propeptide; P1NP, procollagen type 1 amino terminal propeptide; PTH, parathyroid hormone; PYR, pyridinoline; RANK, receptor activator of nuclear factor kappa beta; RANKL, receptor activator of nuclear factor kappa beta ligand; S-, serum; Scl, sclerostin; T1D, type 1 diabetes; T2D, type 2 diabetes; TRAP, tartrate-resistant acid phosphatase; U-, urine; ucOC, undercarboxylated osteocalcin.
T2D compared to controls (Vestergaard, 2007). Despite the higher $\mathrm{BMD}$, patients with T2D have more fractures than non-diabetic controls (Vestergaard, 2007). Patients with T1D also have more hip fractures than can be explained by the decreased BMD (Vestergaard, 2007). The increased fracture risk is supported by a larger Danish study, where diabetics without late complications had a relative risk of any fracture of $1.21(1.07-1.36)$ for T1D and of 1.13 (1.06-1.22) for T2D (Vestergaard et al., 2009). This may point at a weakening of bone biomechanical competence beyond what can be measured by BMD. This disruption of biomechanical competence may be brought about by alterations in bone turnover and non-calcium bone matrix (such as collagen), as BMD mainly reflects calcium content in the bone.

\section{BONE TURNOVER IN GENERAL}

Bone turnover is a dual relationship between the process of bone formation by osteoblasts (creation of new bone) and the process of bone resorption by osteoclasts (removal of old bone) (Delmas, 1991; Garnero, 2009). Bone markers are subdivided into bone formation and bone resorption markers. Bone formation markers consist of osteocalcin (OC), bone-specific alkaline phosphatase (BAP), alkaline phosphatase (AP), osteoprotegerin (OPG), procollagen type 1 amino terminal propeptide (P1NP), and procollagen type 1 carboxyl terminal propeptide (P1CP) 
(Delmas, 1991; Garnero, 2009), while resorptive markers consist of N-terminal cross-linked telopeptide of type-I collagen (NTX), C-terminal cross-linked telopeptide of type-I collagen (CTX), tartrate-resistant acid phosphatase (TRAP), RANKL (Receptor Activator of Nuclear factor Kappa beta Ligand), pyridinoline (PYR), deoxypyridinoline (DPD), hydroxyproline (HP), and sclerostin (Scl) (Delmas, 1991; Manolagas and Almeida, 2007; Garnero, 2009). Scl, OPG, and RANKL are not markers in strict sense, but are included in this section, since they are related to bone turnover (see below). These markers represent products secreted by cells such as OC and Scl and enzymes (alkaline and acid phosphatase), collagen cleavage products as examples of the organic matrix, and calcium itself. Figure 1 gives an overview of some of the different systems (described below) regulating bone turnover.

\section{BIOCHEMICAL MARKERS SECRETED BY BONE CELLS}

(a) Osteocalcin is a valid marker of bone turnover both when formation and resorption are uncoupled as well as when formation and resorption are coupled (Delmas, 1991). However, OC fluctuates with food intake making it susceptible to error and it may be questioned if the serum marker fully reflects what is going on in the bone in diabetics. OC becomes $\gamma$-carboxylated at three glutamine terminals so it can interact with hydroxyapatite, when fewer than three terminals are $\gamma$-carboxylated it is undercarboxylated OC (ucOC) (Motyl et al., 2010). Besides being a marker of bone formation, OC and UcOC are also associated with beta-cell function and insulin sensitivity and thus control of plasma glucose levels (Lee et al., 2007; Ferron et al., 2008; Confavreux et al., 2009). $\mathrm{UcOC}$ is suggested to stimulate the secretion of adiponectin from adipocytes (Motyl et al., 2010; Ng, 2011). In mice, OC injections prevent T2D and improve glucose control (Ferron et al., 2012). Furthermore, a human study concluded that OC is associated with improved glucose tolerance and insulin secretion (Hwang et al., 2012). Glycemic control has been linked to increased OC (Motyl et al., 2010; Bao et al., 2011), although the mechanisms are unclear. (b) Both AP and BAP are bone formation markers measured in serum. While BAP is specific for bone, AP consists of different iso-enzymes like the hepatic AP (Delmas, 1991). (c) TRAP is an enzyme in different subtypes including an osteoclast specific enzyme (TRAP-5b) (Garnero, 2009). TRAP-5b is a marker that reflects the number and activity of osteoclasts and shows little variation regarding time of day and food intake (Garnero, 2009). (d) Scl is a Wnt-pathway antagonist produced in the osteocytes (Gennari et al., 2012). The Wnt-pathway promotes osteoblastogenesis, increases OPG expression, and decreases osteoclastogenesis and bone resorption (Manolagas and Almeida, 2007).

\section{BIOCHEMICAL MARKERS OF COLLAGEN}

P1NP and P1CP are secreted to the blood after their cleavage from collagen (Delmas, 1991). Both P1NP and CTX fluctuate rapidly with glucose intake (Clowes et al., 2003), and may thus be less precise. The rapid fluctuations with glucose intake make it questionable if these markers truly reflect what is going on in the bone during hyperglycemia. PYR is found in collagen of bone, but also in other tissues, while DPD is only at significant amounts in bone collagen (Delmas, 1991). PYR and DPD are mature collagen crosslinks released by collagen breakdown and is thus measurable markers (Calvo et al., 1996). Some of these crosslinks are released in peptide-bound forms (CTX, NTX), which are shown useful in bone assessment (Calvo et al., 1996; Achemlal et al., 2005).

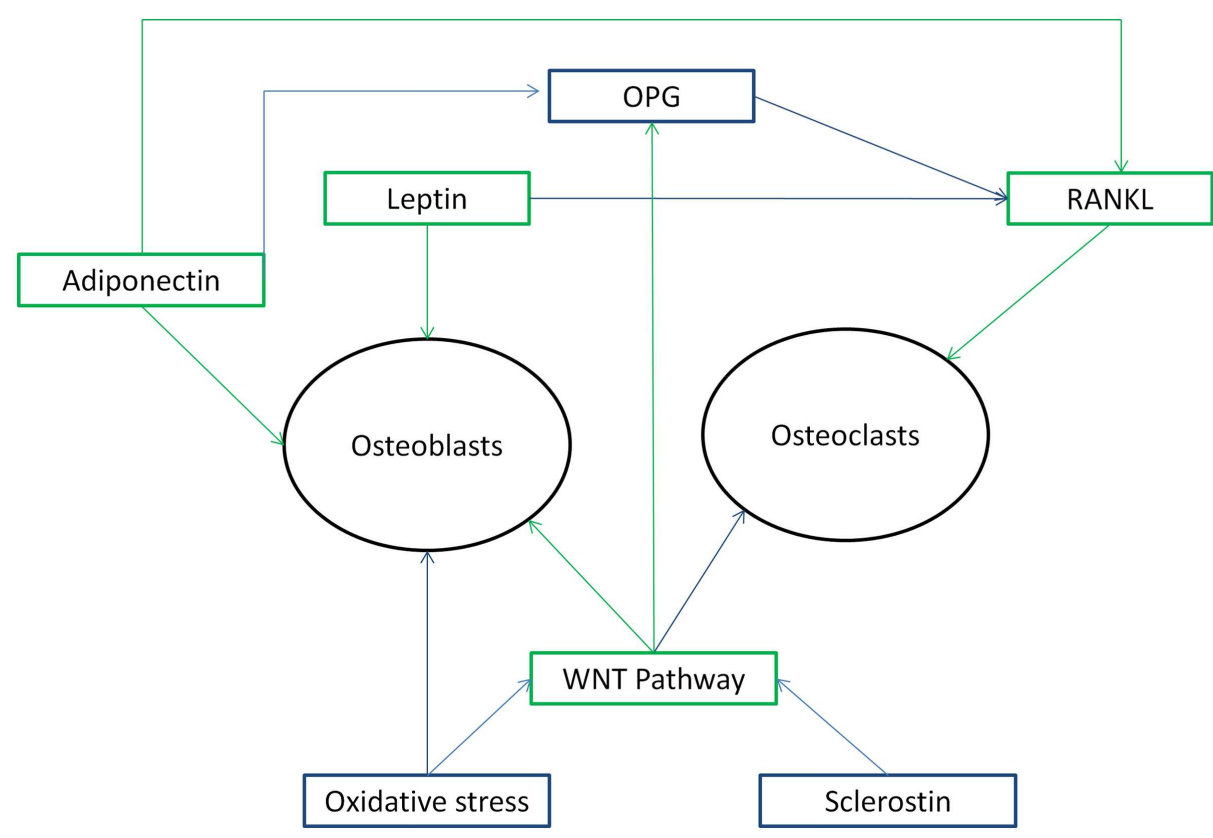

FIGURE 1 | Overview of different systems regulating bone metabolism in its relationship with each other and effects on osteoblasts and osteoclasts as mentioned in the Section "Introduction" above. Figure 1 shows that the systems regulating bone metabolism are in a complex relationship to each other. Green arrows indicate a stimulating action, while blue arrows indicate a inhibiting action. 
Since DPD is a limited marker, it seems more specific and sensitive regarding bone resorption than PYR (Miazgowski and Czekalski, 1998). HP is found in collagens of different tissues and after its release, during collagen breakdown, most of it is reabsorbed in the kidneys and oxidized in the liver. HP thus does not reflect the total collagen catabolism (Delmas, 1991).

\section{CALCIUM}

Serum (s-) calcium is tightly regulated by the calcitropic hormones (see below) and thus often is a poor marker of bone turnover. Urinary (u-) calcium may be used as a marker of bone resorption, but is rather variable with calcium intake and thus requires standardized sampling. For these reasons it is rarely used as a marker of bone turnover in clinical practice. In diabetics, hyperglycemia may be associated with increased loss of calcium in the urine (Raskin et al., 1978; McNair et al., 1979).

\section{CALCITROPIC HORMONES}

The calcium, PTH, and vitamin D system is tightly related to bone metabolism. PTH is secreted from the parathyroid glands and is a regulator of $1,25 \mathrm{OHD}$ production, calcium absorption, and bone calcium release. PTH inhibits Scl production in humans (Garcia-Martin et al., 2012a), thereby influencing bone formation by multiple pathways. Vitamin D acts by stimulating the intestinal absorption of calcium and is a regulator of the calcium homeostasis. Vitamin D stimulates the synthesis of OC (Inaba et al., 1999). Calcitonin affects osteoclasts by inhibiting bone resorption and affects the kidney by enhancing urinary calcium excretion (Pondel, 2000).

\section{THE OPG/RANKL SYSTEM}

The OPG/RANKL system is a part of the bone turnover metabolism. RANKL is the agonist in regulating important aspects of osteoclasts like differentiation, fusion, survival, activation, and apoptosis (Horowitz et al., 2001). RANKL acts by activating a specific receptor (RANK - Receptor Activator of Nuclear factor Kappa beta) on the osteoclasts and promoting bone resorption, making RANKL a marker of bone resorption (Horowitz et al., 2001). In contrast, OPG is the antagonist to RANKL (Galluzzi et al., 2005). Another inhibitor of RANKL seems to be hyperglycemia, which induces low bone turnover and suppresses osteoclastogenesis (Wittrant et al., 2008). Again the question is whether the serum markers truly reflect what is going on in the bone as no biopsy studies are available.

\section{BONE-FAT INTERACTION}

Fat tissue is suggested to be an actor in both diabetes and bone metabolism. T2D patients are often overweight and present with issues related to this (American Diabetes Association, 2012).

(a) Adiponectin is secreted by fat tissue, and a receptor for adiponectin on osteoblasts has been identified (Berner et al., 2004; Kanazawa et al., 2007). Adiponectin is suggested to stimulate proliferation, differentiation, and mineralization of osteoblasts (Berner et al., 2004; Luo et al., 2005; Kanazawa et al., 2007). It is proposed that adiponectin plays a role in energy metabolism by increasing insulin sensitivity, reducing endogenous glucose production, and decreasing serum glucose levels (Berg et al.,
2001; Combs et al., 2001). Adiponectin affects the RANKL/OPG system by stimulating RANKL and inhibiting OPG production in osteoblasts, thereby promoting osteoclast formation and bone resorption (Luo et al., 2006). (b) Another fat tissue marker, leptin, is found to suppress osteoclast and bone resorption in vitro by inhibiting RANKL and may increase bone mass (Holloway et al., 2002; Reid, 2002). Leptin also increases proliferation and differentiation of osteoblasts (Reid, 2002).

\section{THE IGF SYSTEM AND ADVANCED GLYCATION END PRODUCTS}

Another aspect of bone turnover is the effect of insulin and insulin like products. Insulin like growth factor-1 (IGF-1) is an important anabolic regulator of the bone, which mainly is produced in the liver but other tissues as well like in the osteoblasts (Verhaeghe et al., 1992; Johansson et al., 1996; Yakar et al., 2002). In T2D, IGF levels may be increased (Frystyk et al., 1999). In addition insulin is believed to promote osteoblastogenesis and increase bone formation (Reid et al., 1993; Reid, 2002). In contrast, oxidative stress and the production of advanced glycation end products (AGE) are associated with inhibition of osteoblast differentiation and to osteoblast apoptosis, thus leading to reduced osteoblast function (Alikhani et al., 2007; Hamada et al., 2009). Oxidative stress also antagonizes the before mentioned Wnt-pathway and therefore inhibits osteoblast activity (Manolagas and Almeida, 2007).

\section{AIM}

The aim of the present review is to:

(a) Examine in vivo human evidence on the connection between diabetes mellitus and bone markers compared to nondiabetics and collate the different markers with each other.

(b) Evaluate the effect of glycemic control in diabetics on bone markers and assess the similarities and differences between type 1- and type 2-diabetics.

\section{METHODS}

To perform this review a literature search was conducted in association with a research librarian. The databases, PubMed, Embase, Cinahl, SveMed+, Cochrane library, and Bibliotek.dk were screened using the search terms: "Diabetes mellitus," "Diabetes mellitus type 1," "Insulin dependent diabetes (IDD) mellitus," "Diabetes mellitus type 2," "Non-insulin dependent diabetes (NIDD) mellitus," "Bone," "Bone and Bones," "Bone diseases," "Bone turnover," "Hemoglobin A Glycosylated," and "HbA1C." In total 1,188 records were retrieved from the literature search. Duplicates were removed and records screened by title and abstract. The records were screened so they are of a cross-sectional, retrospective, case-control, or prospective design. The eligibility criteria to the studies are; that they shall examine bone turnover markers in relationship to diabetics with or without a control group. Studies assessing the effect of different medications were excluded. By these criteria 1,113 records were removed. The remaining 75 records were assessed in full text for inclusion in the review by the same eligibility criteria as above. In the end 43 records were included in this review. Of the 43 records; 32 were of a cross-sectional design, and 11 were of a prospective design.

Some of the records did not subdivide diabetics in T1D and in T2D, but rather subdivided as IDD and NIDD. The IDD and NIDD 
subdivision is mainly used in studies of older date (all before year 2000). In the following, IDD will be treated as T1D and NIDD will be treated as T2D. However insulin treated T2D may be included in IDD, while NIDD cannot be T1D (which is an IDD type). This may be a setback, still only four studies used IDD as diabetes subdivision (see Tables 1-4), which makes the usage of T1D and IDD as one, a minor issue to the review. Furthermore when glycemic control and 7 years of insulin therapy are mentioned it means a significant decrease in HbAlc or a decrease in FPG. The study using FPG do not conclude whether the drop is significant (Gregorio et al., 1994).

\section{DATA ON MARKERS}

Table 1 shows details on the calcitropic system in diabetics, Table 2 shows details on bone formation markers in diabetics, Table 3 shows details on bone resorption markers in diabetics, and Table 4 shows details on other bone markers in diabetics. An overview of the markers that seem to differ in diabetics is shown in Figure 2.

\section{CALCIUM}

For data on s-calcium and u-calcium, see Table 1. In summary, $\mathrm{s}$-calcium and $\mathrm{u}$-calcium seem not to differ between either T1D or T2D and controls. S-calcium is higher in T2D women than men, with evidence from one study that this may be caused by their postmenopausal state (Rasul et al., 2012a), while another was not informative on this (Pedrazzoni et al., 1989). S-calcium may show a small but significant increase in T2D (2.1 vs. $2.4 \mathrm{mmol} / \mathrm{l}$ ) (Hamilton et al., 2012) over time and poor glycemic control may result in a fall in u-calcium.

\section{PARATHYROID HORMONE}

For data on s-PTH, see Table 1. It is unlikely that renal dysfunction has affected the results, since one study adjusted by creatinine clearance (Dobnig et al., 2006), while all others, expect one (Gerdhem et al., 2005), excluded participants with renal impairment. In summary, s-PTH is likely to be variable in T1D and T2D, since it has been reported to be unchanged, higher, and lower. In T2D the absence of a difference is most likely as it was found by the majority of studies. S-PTH seems not to correlate to BMD in T1D or T2D nor is it likely to differ over time in T1D and T2D, although Vitamin D stimulation decreases s-PTH. Glycemic control is, in T1D, likely to result in a rather large increase in s-PTH, while glycemic control in T2D most likely does not change s-PTH.

\section{SERUM 1,25 VITAMIN D AND 25 VITAMIN D}

For data regarding 1,25 vitamin D and 25 vitamin D, see Table 1 . To summarize S-25OHD is likely to be lower in T1D than controls, while both s-25OHD and s-1,250HD are most likely not to differ between T2D and controls, since the majority of studies reported no difference. S-25OHD may decrease over time in T2D, but not in T1D. The lower s-25OHD levels in T2D may be due to an increased mean age of these individuals (Hamilton et al., 2012). Furthermore glycemic control seems not change s-25OHD in T2D.

\section{CALCITONIN}

For data on calcitonin, see Table 1. In summary, calcitonin seems higher in T1D $(P>0.05)$ than controls, although it is in normal value range, and not to differ in T2D in comparison to controls. However, the number of studies is limited.

\section{ALKALINE PHOSPHATASE}

For data on s-AP, see Table 2. To sum up s-AP seems not to differ in T1D and T2D in comparison to controls, since the largest studies showed no difference (Oz et al., 2006; Zhou et al., 2010; Hamed et al., 2011; Shu et al., 2012). Over time s-AP may increase in T1D, which can be caused by hepatic involvement (Hamilton et al., 2012), since other bone markers (s-OC, s-CTX, u-PYR, uDPD) do not differ. S-AP is also reported not to differ in T1D and T2D over time, nor to change by glycemic control in T1D.

\section{BONE-SPECIFIC ALKALINE PHOSPHATASE}

For data on s-BAP, see Table 2. In summary, s-BAP is most likely not to differ in either T1D or T2D in comparison to controls. S-BAP seems lower in T2D males than T2D females, which may reflect the postmenopausal state in the females (Kanazawa et al., 2011b). S-BAP may not correlate to HbAlc or change over time in T2D, nor is it likely to change by glycemic control in both T1D and T2D.

\section{OSTEOCALCIN}

For data on s-OC, see Table 2. In summary, s-OC is likely to be up to four times lower in young T1D than controls (12.2 vs. $49.4 \mathrm{ng} / \mathrm{ml}$ ) (Abd El Dayem et al., 2011) and somewhat lower in older T1D than controls. A negative relationship to pubertal development is probable in $\mathrm{T} 1 \mathrm{D}$, whereas s-OC may normalize in adulthood. S-OC is likely not to correlate to BMD in T1D, but to have a positive relationship to s-CTX and a negative relationship to HbAlc. In T2D s-OC is most likely to be somewhat lower than among controls, as the studies reporting a lower sOC includes larger populations. Also s-OC is probably negatively associated with HbA1c in T2D. Regarding the longitudinal studies; s-OC is most likely not to change in T1D and T2D over time, while glycemic control neither seem to change s-OC in T1D. However, in T2D, glycemic control may either not change, decrease, or increase s-OC, where the studies finding a decrease were the ones including the longest period of time and therefore supporting a decrease. Overall, changes in s-OC are likely to relate to changes in $\mathrm{HbAlc}$.

\section{UNDERCARBOXYLATED OSTEOCALCIN}

For data on s-ucOC, see Table 2. To sum up, s-ucOC seems twice as low in diabetics on hemodialysis (14.4 vs. $31.5 \mathrm{ng} / \mathrm{ml}$ ) (Okuno et al., 2013) than matched controls and almost half the level in T2D males compared females, which may be explained by the postmenopausal state in the females. S-ucOC appears positively related to bone turnover markers and negatively to $\mathrm{HbA1c}$, while glycemic control does not to change ucOC.

\section{PROCOLLAGEN TYPE 1 AMINO TERMINAL PROPEPTIDE, PROCOLLAGEN TYPE 1 C PROPEPTIDE, AND COLLAGEN TYPE 1 C PROPEPTIDE}

For data on s-P1NP, s-PICP, and s-CICP, see Table 2. To summarize, s-P1NP is likely to be somewhat lower in T2D than controls (see Table 2). On the other hand, neither s-PICP nor s-CICP, are likely to differ regarding T2D, while s-CICP may be somewhat 
Table 1 | Calcitropic system markers.

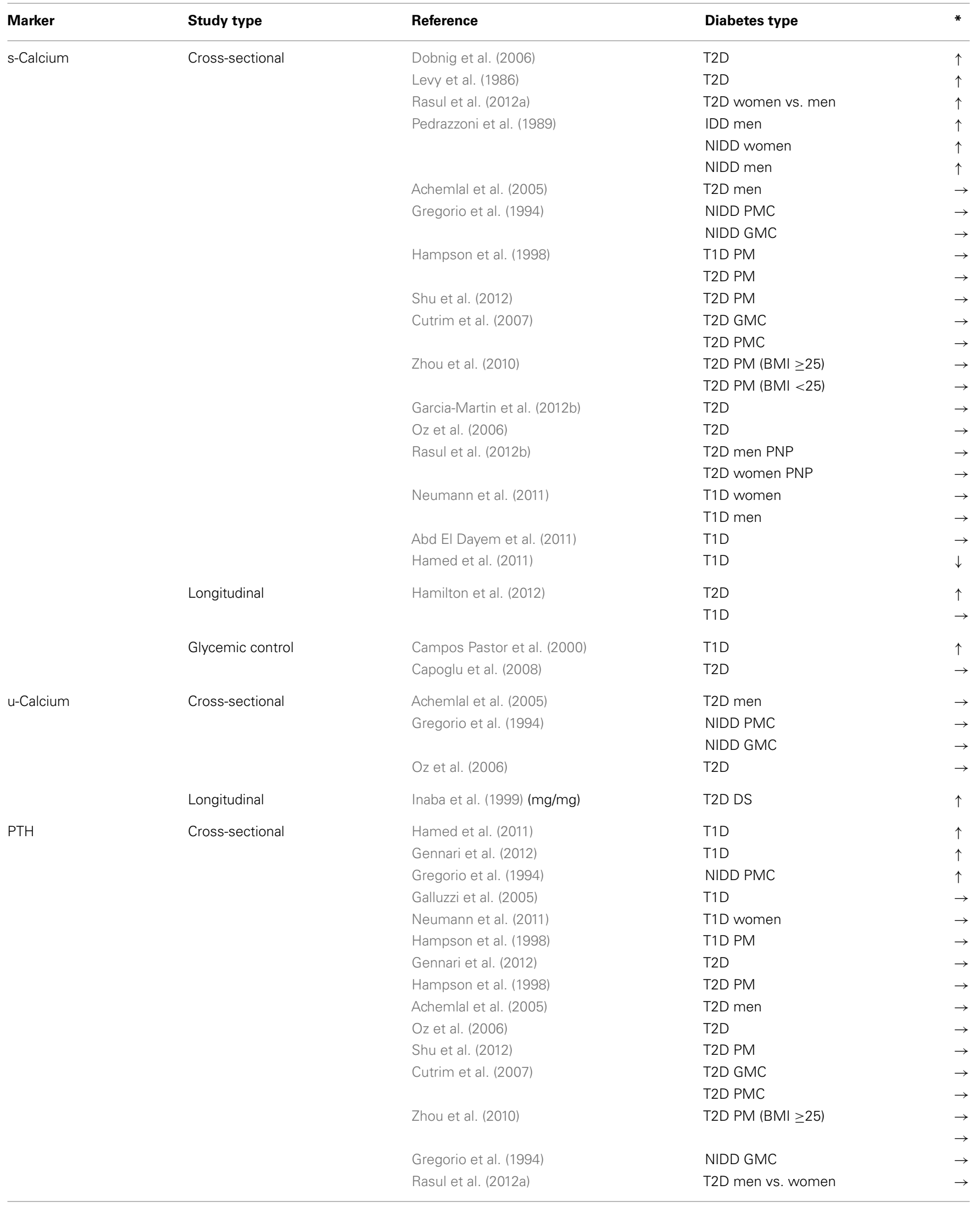


Table 1 | Continued

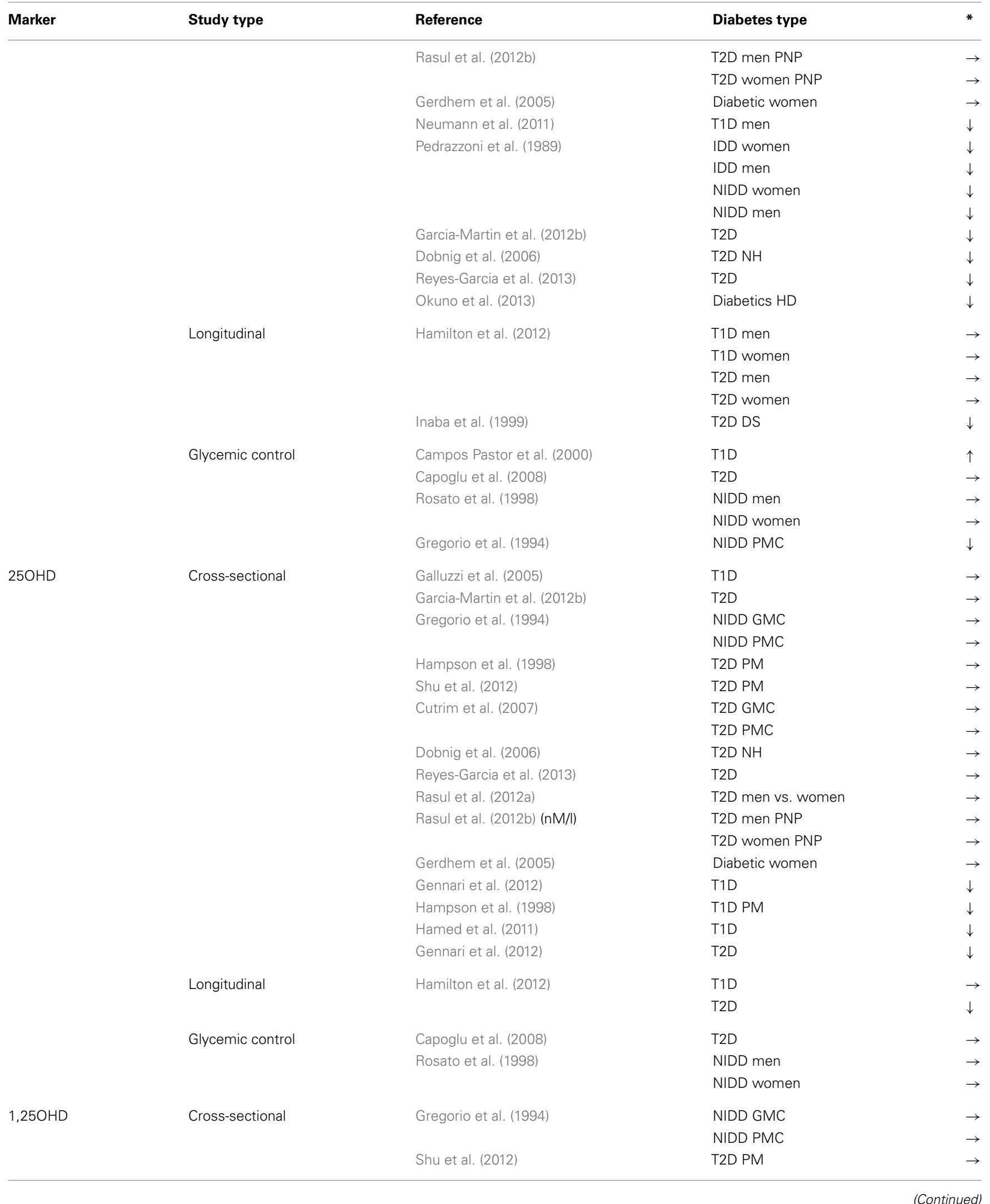


Table 1 | Continued

\begin{tabular}{|c|c|c|c|c|}
\hline Marker & Study type & Reference & Diabetes type & $*$ \\
\hline & & Rasul et al. (2012b) & T2D men PNP & $\rightarrow$ \\
\hline & & & T2D women PNP & $\rightarrow$ \\
\hline & & Rasul et al. (2012a) & T2D men vs. women & $\rightarrow$ \\
\hline \multirow[t]{8}{*}{ Calcitonin } & Cross-sectional & Pedrazzoni et al. (1989) & IDD women & $\uparrow$ \\
\hline & & & IDD men & $\uparrow$ \\
\hline & & & NIDD women & $\uparrow$ \\
\hline & & & NIDD men & $\uparrow$ \\
\hline & & Gregorio et al. (1994) & NIDD GMC & $\rightarrow$ \\
\hline & & & NIDD PMC & $\rightarrow$ \\
\hline & & Zhou et al. (2010) & T2D PM (BMI $\geq 25)$ & $\rightarrow$ \\
\hline & & & T2D PM (BMI <25) & $\rightarrow$ \\
\hline
\end{tabular}

Glycemic control (during the longitudinal study); $*$, statistically significantly different; $\uparrow$, significantly higher in diabetics; $\downarrow$, significantly lower in diabetics; $\rightarrow$, without significance; GMC, good metabolic control; PMC, poor metabolic control; PM, postmenopausal; NH, nursing home; age $\geq 70 ;$ HD, hemodialysis; PNP, polyneuropathy.

lower in T1D supported by a larger study population (see Table 2). $\mathrm{S}-\mathrm{CICP}$ is also probable to be negatively correlated to BMD in T1D.

\section{TARTRATE-RESISTANT ACID PHOSPHATASE}

For data on s-TRAP, see Table 3. S-TRAP-5b is likely to be much lower in T2D than controls. Also s-TRAP seems not to correlate to HbAlc; however it seems to undergo a somewhat large decrease during glycemic control in T1D.

\section{CARBOXY-TERMINAL TELOPEPTIDE OF TYPE 1 COLLAGEN}

For data on s-CTX, see Table 3. In summary, s-CTX is very likely to be up to twice as low in T2D and T1D, when both are compared to controls, since both are reported with convincing significance $(P<0.001)$. Over time s-CTX seems to increase twofold in comparison to the baseline value $(0.26$ vs. $0.13 \mathrm{ng} / \mathrm{ml})$ in T2D females, but not in T2D males or T1D, which could be caused by the probable postmenopausal state due to higher mean age in these T2D females (70 at follow up), while T1D females had a lower mean age (53 at follow up) (Hamilton et al., 2012).

\section{N-TELOPEPTIDE}

For data on N-telopeptide, see Table 3. To summarize, s-NTX is unlikely to differ in either T2D or T1D, but seems to have a negative association with HbA1c in T1D. However, few studies are available.

\section{URINARY N-TERMINAL CROSS-LINKED TELOPEPTIDE OF TYPE-I COLLAGEN}

For data on u-NTX, see Table 3. To sum up, u-NTX is likely to be higher in T2D than controls and be almost twice as low in T2D men as women (see Table 3 ), which may relate to the fact that these women are postmenopausal (Kanazawa et al., 2011b). U-NTX is in T2D likely to correlate negatively to $\mathrm{BMD}$, but not to correlate to HbAlc. Over time, u-NTX does not seem to change in T1D. During glycemic control through 12 months T2D may decrease in u-NTX (Capoglu et al., 2008), while shorter periods do not seem to affect u-NTX.

\section{DEOXYPYRIDINOLINE, PYRIDINOLINE}

For data on u-DPD and u-PYR, see Table 3. In summary, u-DPD may be twice the value in young T1D as controls (Abd El Dayem et al., 2011), while it seems to normalize in adulthood. In T2D, $\mathrm{u}$-DPD is likely not to differ, although unspecified diabetics seem to have lower $\mathrm{u}$-DPD than controls. U-DPD seems not to correlate to BMD in T1D. Nor is u-DPD likely to change over time in T1D and T2D as well as u-PYR does not change in T1D. Furthermore, during glycemic control $\mathrm{u}-\mathrm{PYR}$ seems to increase in T2D, while $\mathrm{u}-\mathrm{DPD}$, on the other hand, is likely to decrease.

\section{HYDROXYPROLINE AND CROSSLAPS}

For data on $\mathrm{u}-\mathrm{HP}$ and $\mathrm{u}$-crosslaps, see Table 3. In summary, $\mathrm{u}$ crosslaps is likely to be lower in T2D than controls. U-HP seems to be positively associated with HbAlc (Gregorio et al., 1994).

\section{OSTEOPROTEGERIN AND RANKL}

For data on OPG and RANKL, see Table 4. In summary s-RANKL is likely not to differ in T1D, while s-OPG may be lower in T1D, since this is supported by a larger study.

\section{SCLEROSTIN}

For data on s-Scl, see Table 4. Also, s-Scl tended to correlate positively to HbA1c levels in T2D patients, however the correlation was not statistically significant (Garcia-Martin et al., 2012b; Gennari et al., 2012). In relation to BMD, s-Scl is in T2D reported to positively relate to $T$-score in lumbar spine, femoral neck, and total hip $(P<0.05)$ (Garcia-Martin et al., 2012b) and s-Scl levels are lower in osteoporotic- than non-osteoporotic-T2D $(P=0.048)$ (Garcia-Martin et al., 2012b). In summary, s-Scl is likely to be higher in T2D than both T1D and controls. S-Scl tends to be positively related to $\mathrm{HbAlc}$ and is likely to be positively related to BMD in T2D.

\section{ADIPONECTIN AND LEPTIN}

For data on adiponectin, see Table 4. In summary, s-adiponectin is likely to be higher in T2D women than T2D men, which may be due to postmenopausal state among the women studied (Kanazawa 
Table 2 | Bone formation markers.

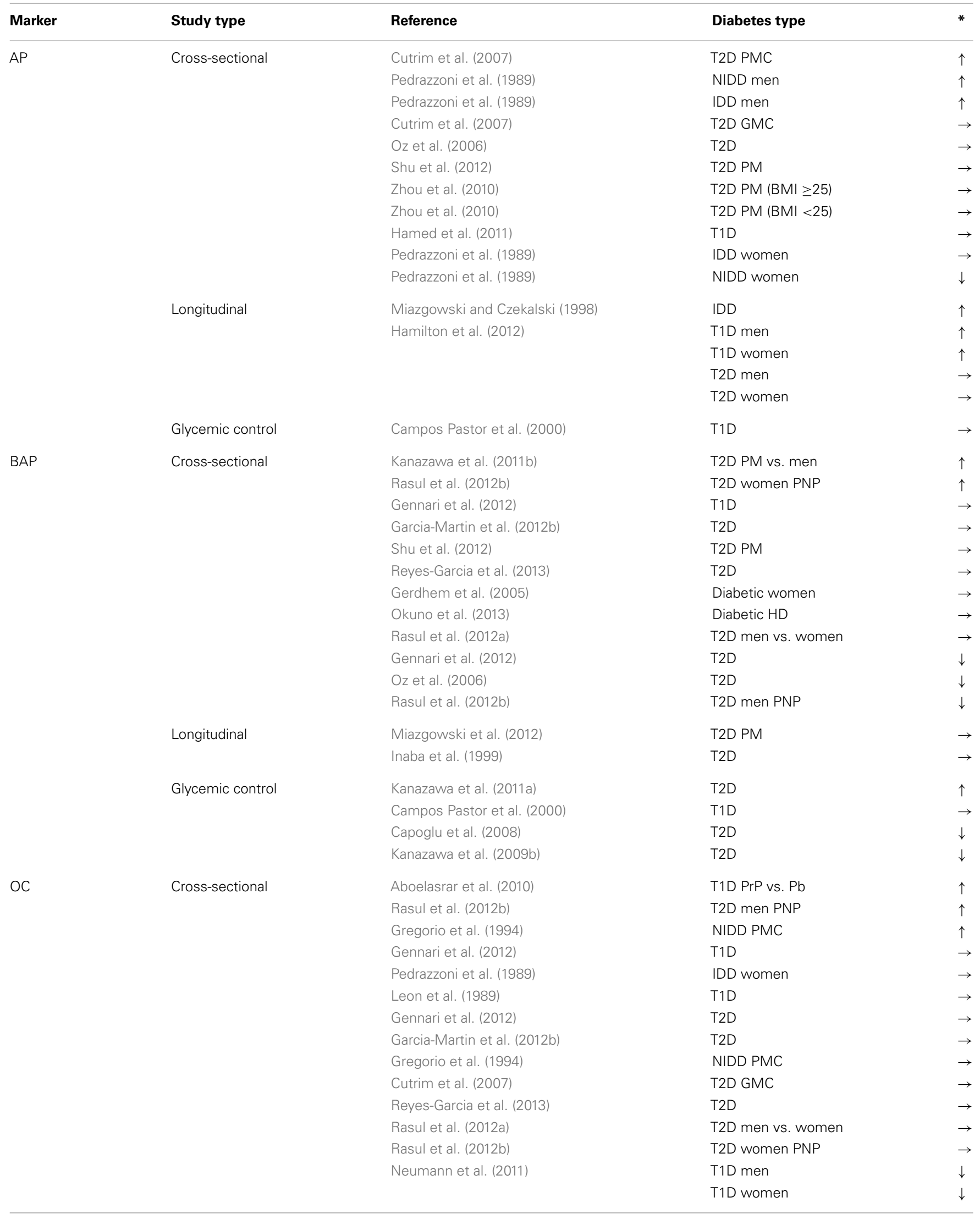


Table 2 | Continued

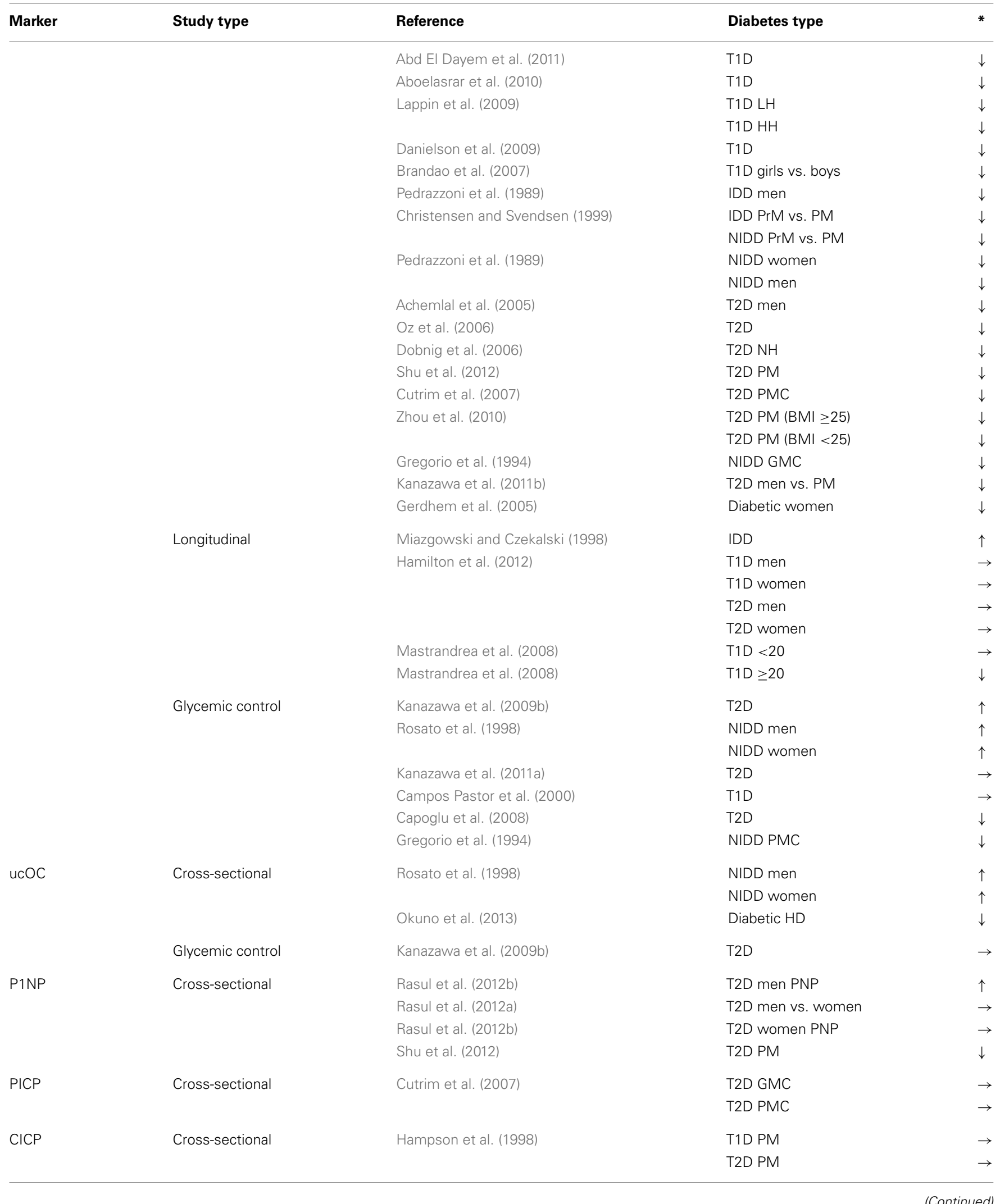

(Continued) 


\begin{tabular}{|c|c|c|c|c|}
\hline Marker & Study type & Reference & Diabetes type & $*$ \\
\hline & & Oz et al. (2006) & $\mathrm{T} 2 \mathrm{D}$ & $\rightarrow$ \\
\hline & & Lappin et al. (2009) & T1D LH & $\rightarrow$ \\
\hline & & & T1D HH & $\rightarrow$ \\
\hline & & Abd El Dayem et al. (2011) & $\mathrm{T} 1 \mathrm{D}$ & $\downarrow$ \\
\hline
\end{tabular}

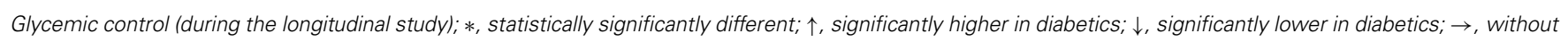
significance; GMC, good metabolic control; PMC, poor metabolic control; PM, postmenopausal; NH, nursing home; age $\geq 70 ;$ HD, hemodialysis; PNP, polyneuropathy; PrM, premenopausal; LH, low HbA1c <8.5; HH, high HbA1c >8.5; 220,20 years old or more; <20, younger than 20 years; Pb, pubertal; PrP, prepubertal.

et al., 2009a). It is uncertain whether s-adiponectin is related to bone turnover markers, while s-leptin seems to have a negative relationship to u-NTX. Moreover glycemic control seems not to change s-adiponectin in T2D.

\section{IGF-1}

For data on s-IGF, see Table 4. To summarize, s-IGF-1 is likely to be somewhat lower in T1D and not to differ in T2D. Over time, s-IGF-1 seems to decrease in younger T1D, while it did not differ in older T1D. This may be caused by a depletion of the insulin function in the young T1D at follow up. Also, during glycemic control IGF-1 seems to increase in T2D.

\section{DISCUSSION}

In general a major issue is the few histomorphometric studies in humans (Leite Duarte and da Silva, 1996; Armas et al., 2012), and the absence of studies on the association between biochemical markers of bone turnover in blood and actual changes in bone tissue. Several markers, especially OC, CTX, and P1NP may also vary with blood glucose or glucose intake, making them perhaps less markers of bone turnover in diabetics and more markers of alterations in glucose metabolism. Another issue is kidney function, which may influence the measurement of several biochemical markers of bone turnover and also influence histomorphometry of the bone (Andress et al., 1987).

\section{TYPE 1 DIABETICS VS. NON-DIABETICS}

Neither s-calcium nor $\mathrm{u}$-calcium seems to be specific markers of bone in T1D or differ in comparison to controls (Hampson et al., 1998; Brandao et al., 2007; Abd El Dayem et al., 2011; Neumann et al., 2011), although young T1D might have a lower s-calcium (Hamed et al., 2011). Hence may the bone deficiency, by lower BMD and increased fracture risk (Vestergaard, 2007; Vestergaard et al., 2009) in T1D, be formed in childhood, leaving the bones fragile in adulthood, while calcium levels normalize.

Regarding bone turnover markers secreted by bone cells; s-AP, s-BAP, and s-Scl seem not to differ in T1D compared to controls (Leon et al., 1989; Munoz-Torres et al., 1996; Oz et al., 2006; Hamed et al., 2011; Gennari et al., 2012), and s-TRAP seems in normal value range (Munoz-Torres et al., 1996). SOC appear to be lower in T1D and may decrease during the pubertal growth (Brandao et al., 2007; Aboelasrar et al., 2010; Abd El Dayem et al., 2011); so those diagnosed before adulthood are bone growth impaired and the bone affection may continue in adulthood (Danielson et al., 2009; Lappin et al., 2009). The lack of an association of s-OC with BMD (Munoz-Torres et al., 1996; Brandao et al., 2007; Danielson et al., 2009) and the positive relationship to s-CTX (Brandao et al., 2007; Abd El Dayem et al., 2011) suggest that formation and resorption are coupled processes in $\mathrm{T} 1 \mathrm{D}$, and may not affect BMD. The negative associations of s-OC to HbAlc (Danielson et al., 2009; Aboelasrar et al., 2010; Abd El Dayem et al., 2011) suggest that bone formation is impaired by high levels of blood glucose; accordingly T1D with poor glycemic control has more fragile bones than T1D with good glycemic control.

When looking at bone turnover markers of collagen; s-CTX and s-CICP are likely to be lower in T1D (Abd El Dayem et al., 2011; Neumann et al., 2011; Gennari et al., 2012) and u-DPD may be higher in young T1D (Abd El Dayem et al., 2011), while $\mathrm{u}$-crosslaps and s-NTX do not differ (Christensen and Svendsen, 1999; Danielson et al., 2009). Seemingly s-CICP is raised, while bone resorption markers may not differ, be raised or be lowered, thus making it difficult to conclude definitively. The absence of a correlation of HbA1c and S-CTX (Lappin et al., 2009), and a negative correlation to s-NTX (Danielson et al., 2009) suggest that high HbA1c may affect bone resorption negatively. In young T1D, s-CTX seems negatively related to pubertal development (Brandao et al., 2007), suggesting that during pubertal development bone resorption is impaired in T1D. Taken together with the corresponding finding regarding $\mathrm{s}-\mathrm{OC}$, bone turnover seems to be reduced during pubertal development in T1D. The RANKL/OPG system has only been investigated in T1D, where bone resorption appears to be affected by a reduced OPG. Even so, s-CTX does not relate to s-OPG, but a positive relationship is apparent with s-RANKL and the RANKL/OPG ratio (Lappin et al., 2009). Seemingly, s-CTX is a marker of activity in the RANKL system and might be the end product of the process. Increased blood glucose seems not to be the mechanism that suppress s-OPG, since s-OPG is found positively correlated to HbA1c (Galluzzi et al., 2005; Lappin et al., 2009), indicating that increasing levels of blood glucose inhibit bone resorption.

\section{TYPE 2 DIABETICS VS. NON-DIABETICS}

Neither s-calcium nor u-calcium differed between T2D and controls (Gregorio et al., 1994; Hampson et al., 1998; Achemlal et al., 2005; Oz et al., 2006; Cutrim et al., 2007; Zhou et al., 2010; GarciaMartin et al., 2012b; Shu et al., 2012). S-calcium appears not to correlate to HbAlc or BMD (Levy et al., 1986; Hampson et al., 1998), thus making it a poor marker of bone- and glycemic-status 
Table 3 | Bone resorption markers.

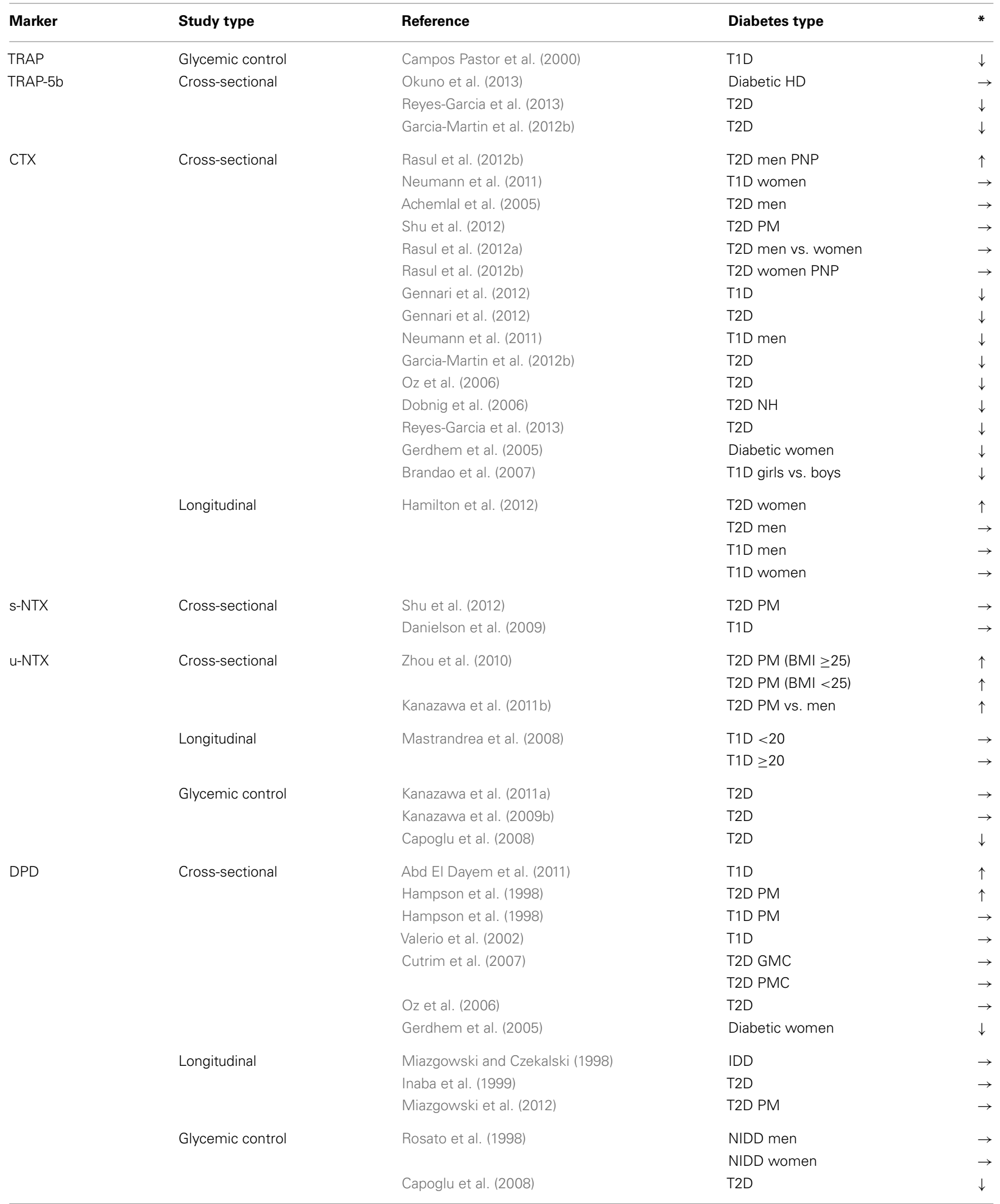


Table 3 | Continued

\begin{tabular}{llll}
\hline Marker & Study type & Reference & Diabetes type \\
\hline PYR & Longitudinal & Miazgowski and Czekalski (1998) & IDD \\
& & Inaba et al. (1999) & T2D \\
& & Nosato et al. (1998) men & NIDD women \\
& & & NIDD w \\
Crosslaps & Cross-sectional & Christensen and Svendsen (1999) & IDD PM vs. PrM \\
& & & NIDD PM vs. PrM \\
HP & Cross-sectional & Gregorio et al. (1994) NIDD PMC & NIDD GMC \\
& & & $\uparrow$ \\
\hline
\end{tabular}

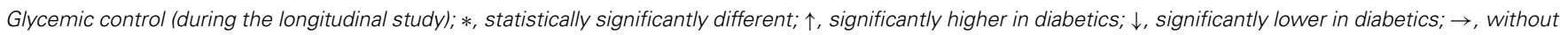

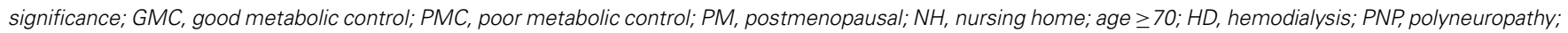
PrM, premenopausal; $\geq 20,20$ years old or more; $<20$, younger than 20 years.

Table 4 | Other markers.

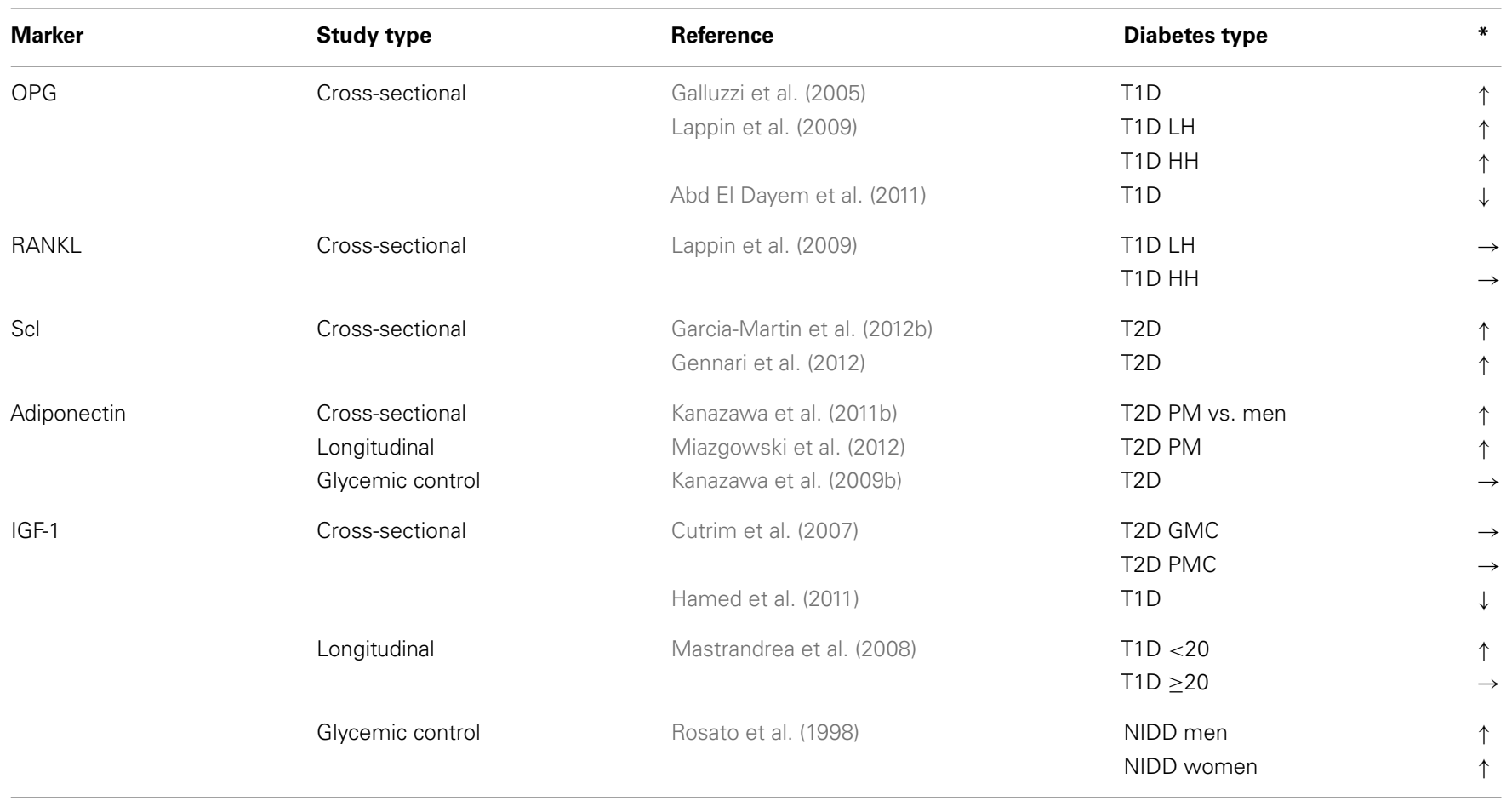

Glycemic control (during the longitudinal study); *, statistically significantly different; $\uparrow$, significantly higher in diabetics; $\downarrow$, significantly lower in diabetics; $\rightarrow$, without significance; GMC, good metabolic control; PMC, poor metabolic control; $P M$, postmenopausal; $L H$, low HbA1C <8.5; HH, high HbA1C >8.5.

in T2D. The PTH-vitamin D axis; S-PTH, 1,25OHD, s-25OHD, and calcitonin are most likely not to be affected in T2D (Pedrazzoni et al., 1989; Gregorio et al., 1994; Hampson et al., 1998; Achemlal et al., 2005; Dobnig et al., 2006; Oz et al., 2006; Cutrim et al., 2007; Zhou et al., 2010; Garcia-Martin et al., 2012b; Gennari et al., 2012; Shu et al., 2012; Reyes-Garcia et al., 2013). The positive relationship between s-PTH and the resorptive markers s-TRAP-5b and s-CTX (Reyes-Garcia et al., 2013) suggests that PTH induce bone resorption in $\mathrm{T} 2 \mathrm{D}$.

Concerning bone turnover markers secreted by bone cells; sAP and s-BAP seem not to differ in T2D (Oz et al., 2006; Zhou et al., 2010; Garcia-Martin et al., 2012b; Shu et al., 2012; ReyesGarcia et al., 2013). S-OC and s-TRAP are likely to be decreased in T2D (Rosato et al., 1998; Achemlal et al., 2005; Dobnig et al., 2006; Oz et al., 2006; Zhou et al., 2010; Garcia-Martin et al., 2012b; Shu et al., 2012; Reyes-Garcia et al., 2013) and s-Scl, which impair bone formation (Manolagas and Almeida, 2007; Gennari et al., 2012), seems increased, suggesting that T2D is in a state of low bone turnover. S-OC seems and s-Scl tends to correlate negatively to HbA1c in T2D (Dobnig et al., 2006; Kanazawa et al., 2009a; Garcia-Martin et al., 2012b; Gennari et al., 2012), thus suggesting that the low bone turnover and bone deficiency in 


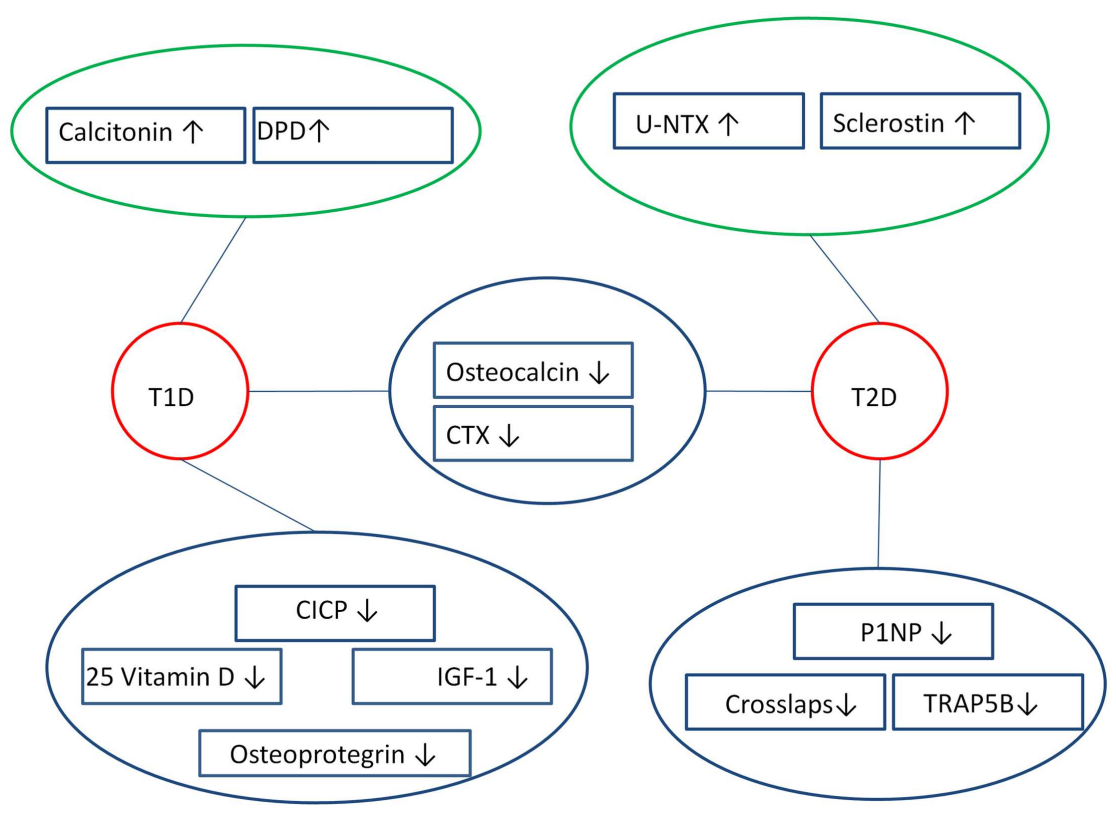

FIGURE 2 | Overview of bone markers that are likely to differ in diabetics compared to controls. Both T1D and T2D appear to have altered bone resorption markers and bone formation markers, where bone formation markers (OC, P1NP, CICP) are lowered, while bone resorption markers (DPD, u-NTX, CTX, TRAP) are either lowered or raised. This reveals a dissociation in the bone markers among diabetics, as bone markers that do not differ are left out of the figure. $\uparrow$ Indicates raised marker, $\downarrow$ indicates lowered marker, blue circle surrounds markers that appear to be lowered, green circle surrounds markers that seem to be raised, red circle surrounds diabetics.
T2D may be caused by elevated glycemic levels. S-BAP have a negative relationship with IGF-1 (Kanazawa et al., 2009a, 2011b), while s-OC is in positive relationship with IGF-1 (Kanazawa et al., 2009a), proposing that IGF-1 is a marker of bone formation in T2D specific to OC. S-BAP, s-TRAP-5b, and s-CTX correlated negatively to s-Scl (Garcia-Martin et al., 2012b; Gennari et al., 2012), thus Scl correlates to decreased bone turnover, while it in contrast correlated to increased BMD (Garcia-Martin et al., 2012b). The increase in BMD is inconsistent with the antagonizing effect on the Wnt-pathway (Manolagas and Almeida, 2007). When looking at bone turnover markers of collagen; u-NTX is higher (Zhou et al., 2010) and s-NTX, s-PICP, s-CICP, and u-DPD seem not to differ (Hampson et al., 1998; Oz et al., 2006; Cutrim et al., 2007; Shu et al., 2012), while u-crosslaps, s-CTX, and s-P1NP are lower (Christensen and Svendsen, 1999; Dobnig et al., 2006; Oz et al., 2006; Garcia-Martin et al., 2012b; Gennari et al., 2012; Shu et al., 2012; Reyes-Garcia et al., 2013), suggesting that bone turnover is changed in T2D. The differences in bone resorption markers may reflect different points of progress in T2D bone affection or the fact that markers may be sensitive or insensitive in T2D. The markers may also be affected by the heterogeneity of the T2D state. Also, S-CTX is negatively related to and P1NP tends to negatively relate to HbA1c (Achemlal et al., 2005; Dobnig et al., 2006; Shu et al., 2012), while u-HP is positively related to HbAlc (Gregorio et al., 1994) and u-NTX does not relate to HbA1c (Kanazawa et al., 2009a). Apparently elevated levels of blood glucose suppress bone formation, and decrease or increase markers of bone resorption in $\mathrm{T} 2 \mathrm{D}$, which is consistent with the findings concerning s-OC and s-Scl. Therefore blood glucose may have taken part in the previous mentioned fluctuating pattern of the resorption markers. The negative relationship between S-CTX, u-NTX, and BMD (Reyes-Garcia et al., 2013), suggest these markers as informants on $\mathrm{BMD}$ and that extensive bone resorption cause low BMD.

Concerning fat tissue hormones; adiponectin and leptin may have a positive effect on bone status by suppressing resorptive markers, increasing bone formation markers and BMD (Tamura et al., 2007; Kanazawa et al., 2011b). However, this is uncertain and further studies are needed.

\section{TYPE 1 VS. TYPE 2 DIABETICS}

The lack of a difference in bone turnover markers indicate that T1D and T2D (Hampson et al., 1998; Gennari et al., 2012) are not different regarding the effect on bone markers, although Scl levels are higher in T2D (Gennari et al., 2012), proposing that bones are affected through an antagonizing effect on the WNT-pathway in T2D, but not in T1D. However the full selection of bone markers is not represented.

\section{HOW SEVERITY OF DISEASE AFFECTS MARKERS}

Severity of disease is characterized by diabetics with polyneuropathy or diabetics in hemodialysis. S-P1NP is reported higher in T2D with polyneuropathy than regular T2D otherwise no markers differ (OC and s-BAP) (Rasul et al., 2012b). In diabetics in hemodialysis s-ucOC and s-PTH are lower, while s-TRAP-5b and s-BAP do not differ in comparison to equally ill controls (Okuno et al., 2013). More severe diabetes is likely to affect bone markers by raising $\mathrm{P} 1 \mathrm{NP}$ and lowering s-ucOC, this may be caused by the severe disease itself. 


\section{DURATION OF DIABETES AND THE EFFECT ON BONE}

S-OC in T1D (Abd El Dayem et al., 2011) and S-TRAP-5b in T2D (Reyes-Garcia et al., 2013), are negatively correlated to the duration of diabetes. This suggests that long term diabetes lead to suppressed bone turnover and thus potentially fragile bones.

\section{THE EFFECT OF TIME ON MARKERS IN TYPE 1 DIABETICS}

The markers s-OC, s-CTX, u-PYR, u-DPD are likely not to differ over time (Miazgowski and Czekalski, 1998; Mastrandrea et al., 2008; Hamilton et al., 2012), suggesting that bone turnover does not change over time in T1D and support the hypothesis that bone turnover is lowered during puberty, since none of the available longitudinal studies have investigated a young T1D population.

\section{THE EFFECT OF TIME ON MARKERS IN TYPE 2 DIABETICS}

The resorption marker s-CTX increase over 5 years, while no other bone turnover marker seems to change (Miazgowski and Czekalski, 1998; Hamilton et al., 2012), suggesting either no change in bone turnover over time, thus the s-CTX increase may be seen by chance, or that s-CTX is a specific marker in T2D, where others are not. An increase in s-25OHD may affect this; even so PTH did not change (Miazgowski and Czekalski, 1998; Hamilton et al., 2012). The s-adiponectin increase before it normalized back to baseline level in newly diagnosed in the study by Miazgowski et al. (2012) suggest, together with a negative relationship to BMD, that a quick rise and following normalization in s-adiponectin may lower BMD in T2D. On the other hand, changes in femoral neck BMD seems to relate positively to baseline log [adiponectin] (Kanazawa et al., 2010), which does not accord to the above. Over time HbAlc does not seem to be related to u-DPD, s-BAP (Kanazawa et al., 2010; Miazgowski et al., 2012), suggesting that HbAlc does not affect bone turnover markers.

\section{THE CHANGES BY GLYCEMIC CONTROL}

Seven years of intensive insulin therapy decrease s-TRAP, while bone formation markers did not change in T1D (Campos Pastor et al., 2000), indicating that glycemic control alters bone resorption, while it has no effect on bone formation.

The intervention of glycemic control is, in T2D, executed by regular diabetes control, diet, exercise, or medical treatment. The glycemic control seems not to change s-BAP, ucOC, s-PTH, s-25OHD, and s-adiponectin, while u-PYR and s-IGF-1 seem to increase, and u-DPD, $\mathrm{u}-\mathrm{HP}, \mathrm{u}-$ calcium, and $\mathrm{u}-\mathrm{NTX}$ seem to decrease (Gregorio et al., 1994; Rosato et al., 1998; Capoglu et al., 2008; Kanazawa et al., 2009b). S-OC may not change, increase, or decrease during glycemic control (Gregorio et al., 1994; Capoglu et al., 2008; Kanazawa et al., 2009b, 2011a), where a short period of glycemic control increase s-OC, intermediate periods decrease sOC, and the longest available period of glycemic control (2 years) increase s-OC (Rosato et al., 1998). The previously proposed positive effect of glycemic control on OC may be present, however the link between OC and glycemic control seems ambiguous due to the different reports. In general; bone resorption may decrease during glycemic control, although u-PYR increase, and bone formation may not change. PYR is not as good a bone marker as DPD, since it is not specific for bone tissue, so to say it is most likely that bone resorption is lowered in T2D, and the PYR increase could be due to collagen breakdown at non-bone sites, suggesting glycemic control to decrease bone resorption.

\section{COHORTS}

The data on biochemical markers was collected from several records as mentioned in the Section "Methods." In general the studies used small cohorts consisting of around 50 diabetics and a similar control group. The studies on T2D have larger cohorts than studies on T1D, whereas the largest T1D cohort is of 128 participants (Neumann et al., 2011), while the largest diabetic cohort by far is found in Zhou et al. (2010) where 890 postmenopausal T2D were examined. Another aspect is that the cohorts were very heterogeneous in age and postmenopausal status among women. Most studies on T2D included have a mean age around 60 and if they included women, they were postmenopausal, although other studies have older or younger populations. In opposition the studies examining T1D primarily looked at younger populations and a larger fraction of the studies assessed bone status in children and/or adolescents. The studies have different means by assessing diabetes in their participant; some use criteria by WHO or American Diabetes Association, while others retrieve the patients from hospitals and outpatient clinics and a study does not mention how they got their diabetic participants (Okuno et al., 2013). However all these methods, except the last mentioned, seem reliable and therefore the results seem to consider diabetics. Another problem in comparing the results of the studies is the difference in diabetes duration. T1D cohorts have been reported with mean diabetes duration varying from 2.67 (Hamed et al., 2011 ) to 18.5 years (Gennari et al., 2012). For T2D the variation in time since diagnosis spans from a diagnosis made within the last year (Miazgowski et al., 2012) to 14.3 years (Tamura et al., 2007). This is an issue, since diabetes duration seems to affect bone turnover markers negatively and therefore makes it hard to compare the studies with different disease durations. Regarding the longitudinal studies; their period of follow up differs from 1 month (Kanazawa et al., 2009b) to 7 years (Campos Pastor et al., 2000), which may question the comparability of the results. Many confounders are also likely to influence the results including co morbidities, diabetes medication, other medications, and baseline characteristics as BMI, smoking, and alcohol. Even though most studies exclude participants with bone metabolism related diseases and bone metabolism affecting treatment their exclusion criteria are not unanimous. Also T2D is in the available records medically treated very differently. Some receive only diet changes, while others receive oral anti diabetics and others again insulin or a mix of different treatment modalities. This is a subject of concern regarding the reliability of the comparability of the different results.

The small cohorts, the heterogeneity among the studies regarding age, menopausal state, diabetes duration, exclusion criteria, and diabetes treatment may affect the results on the bone turnover, whereas truly hidden bone affection may be hidden in the large number of confounders.

\section{CLOSING REMARKS}

The paradox of increased Scl and yet increased BMD in T2D, may be partly explained by inflammation. Inflammation is a part of 
both the T1D and the T2D disease (Bending et al., 2012; Calle and Fernandez, 2012). This may affect the bone markers and Scl as in inflammatory diseases, which is linked to bone resorption and osteoporotic fractures (Lacativa and Farias, 2010). Hence the effect on certain bone markers, as concluded in this review, may be due to inflammation.

The differences in bone turnover markers may also relate to the fact, that some markers are specific in diabetics, while others are not. The specificity of markers could be due to diabetes per se or the results of diabetes by blood glucose alterations, insulin deficiency, and AGE. Elevated blood glucose may result in measurement errors regarding bone markers, which could explain these differences. In addition cohort differences may influence the findings and hide the true effect of diabetes on bone markers by confounders. In experimental rat models, where the human confounders are not present, bone markers as OC, PYR, and TRAP-5b are reported decreased in diabetic rats in comparison to controls (Herrero et al., 1998; Suzuki et al., 1998). Therefore supporting that bone markers and bone turnover are lowered in the diabetic state and the fluctuating pattern in humans may be due to other effects than diabetes per se. However the findings regarding rats may not be transferable to humans. In conclusion the alteration of

\section{REFERENCES}

Abd El Dayem, S. M., El-Shehaby, A. M., Abd El Gafar, A., Fawzy, A., and Salama, H. (2011). Bone density, body composition, and markers of bone remodeling in type 1 diabetic patients. Scand. J. Clin. Lab. Invest. 71, 387-393.

Aboelasrar, M., Farid, S., El Maraghy, M., and Mohamedeen, A. (2010). Serum osteocalcin, zinc nutritive status and bone turnover in children and adolescents with type 1 diabetes mellitus. Pediatr. Diabetes 11, 50.

Achemlal, L., Tellal, S., Rkiouak, F., Nouijai, A., Bezza, A., Derouiche, E. M., et al. (2005). Bone metabolism in male patients with type 2 diabetes. Clin. Rheumatol. 24, 493-496.

Alikhani, M., Alikhani, Z., Boyd, C., MacLellan, C. M., Raptis, M., Liu, R., et al. (2007). Advanced glycation end products stimulate osteoblast apoptosis via the MAP kinase and cytosolic apoptotic pathways. Bone 40, 345-353.

American Diabetes Association. (2012). Diagnosis and classification of diabetes mellitus. Diabetes Care 35(Suppl. 1), S64-S71.

Andress, D. L., Hercz, G., Kopp, J. B., Endres, D. B., Norris, K. C., Coburn, J. W., et al. (1987). Bone histomorphometry of renal osteodystrophy in diabetic patients. J. Bone Miner. Res. 2, 525-531.

Armas, L. A., Akhter, M. P., Drincic, A., and Recker, R. R. (2012). Trabecular bone histomorphometry in humans

bone turnover in T1D and T2D may be mediated through elevated blood glucose levels and long duration of diabetes.

\section{PERSPECTIVES}

In general bone formation and resorption are tightly coupled, and formation markers and resorptive markers tend to change in a coordinated way. The dissociation seen in diabetes, where some markers decrease (both formation markers, such as OC in both T1D and T2D, and resorption markers such as CTX and TRAP in T2D), whereas the remainder do not (e.g., AP) could point to a very specific uncoupling effect on these of factors associated with the disruption in glucose metabolism in diabetes. Perhaps glucose alters the circulating levels without affecting bone turnover per se. This may be supported by the only histomorphometric study in humans with T1D, which showed no alteration in bone turnover (Armas et al., 2012). However, more research is needed, perhaps including modern PET scanning techniques using fluoride to elucidate bone turnover (Puri et al., 2012).

\section{ACKNOWLEDGMENTS}

The author acknowledges the assistance by research librarian Ms. Edith Clausen.

Pardo, M. D., and Garcia-Cervigon, A. G. (2000). Intensive insulin therapy and bone mineral density in type 1 diabetes mellitus: a prospective study. Osteoporos. Int. 11, 455-459.

Capoglu, I., Ozkan, A., Ozkan, B., and Umudum, Z. (2008). Bone turnover markers in patients with type 2 diabetes and their correlation with glycosylated haemoglobin levels. J. Int. Med. Res. 36, 1392-1398.

Christensen, J. O., and Svendsen, O. L. (1999). Bone mineral in pre and postmenopausal women with insulin-dependent and non-insulindependent diabetes mellitus. Osteoporos. Int. 10, 307-311.

Clowes, J. A., Allen, H. C., Prentis, D. M., Eastell, R., and Blumsohn, A. (2003). Octreotide abolishes the acute decrease in bone turnover in response to oral glucose. J. Clin. Endocrinol. Metab. 88, 4867-4873.

Combs, T. P., Berg, A. H., Obici, S., Scherer, P. E., and Rossetti, L. (2001). Endogenous glucose production is inhibited by the adipose-derived protein Acrp30. J. Clin. Invest. 108, 1875-1881.

Confavreux, C. B., Levine, R. L., and Karsenty, G. (2009). A paradigm of integrative physiology, the crosstalk between bone and energy metabolisms. Mol. Cell. Endocrinol. 310, 21-29.

Cutrim, D. M. S. L., Pereira, F. A., de Paula, F. J. A., and Foss, M. C. (2007). Lack of relationship between glycemic control and bone mineral density in type 2 diabetes mellitus. Braz. J. Med. Biol. Res. 40, 221-227.

Danielson, K. K., Elliott, M. E., Lecaire, T., Binkley, N., and Palta, M. (2009). Poor glycemic control is associated with low BMD detected in premenopausal women with type 1 diabetes. Osteoporos. Int. 20, 923-933.

Delmas, P. D. (1991). What do we know about biochemical bone markers? Baillieres Clin. Obstet. Gynaecol. 5, 817-830.

Dobnig, H., Piswanger-Solkner, J. C., Roth, M., Obermayer-Pietsch, B. Tiran, A., Strele, A., et al. (2006). Type 2 diabetes mellitus in nursing home patients: effects on bone turnover, bone mass, and fracture risk. J. Clin. Endocrinol. Metab. 91, 3355-3363.

Ferron, M., Hinoi, E., Karsenty, G., and Ducy, P. (2008). Osteocalcin differentially regulates beta cell and adipocyte gene expression and affects the development of metabolic diseases in wild-type mice. Proc. Natl. Acad. Sci. U.S.A. 105, 5266-5270.

Ferron, M., McKee, M. D., Levine, R. L., Ducy, P., and Karsenty, G. (2012). Intermittent injections of osteocalcin improve glucose metabolism and prevent type 2 diabetes in mice. Bone 50, 568-575.

Frystyk, J., Skjaerbaek, C., Vestbo, E., Fisker, S., and Orskov, H. (1999). Circulating levels of free insulin-like growth factors in obese subjects: the impact of type 2 diabetes. Diabetes Metab. Res. Rev. 15, 314-322. 
Galluzzi, F., Stagi, S., Salti, R., Toni, S., Piscitelli, E., Simonini, G., et al. (2005). Osteoprotegerin serum levels in children with type 1 diabetes: a potential modulating role in bone status. Eur. J. Endocrinol. 153, 879-885.

Garcia-Martin, A., Rozas-Moreno, P., Reyes-Garcia, R., Morales-Santana, S., Garcia-Fontana, B., GarciaSalcedo, J. A., et al. (2012a). Circulating levels of sclerostin are increased in patients with type 2 diabetes mellitus. J. Clin. Endocrinol. Metab. 97, 234-241.

Garcia-Martin, A., Reyes-Garcia, R., Rozas-Moreno, P., Morales-Santana, S., Garcia-Fontana, B., and MunozTorres, M. (2012b). Role of serum sclerostin on bone metabolism in patients with type 2 diabetes mellitus. Osteoporos. Int. 23, S150-S151.

Garnero, P. (2009). Bone markers in osteoporosis. Curr. Osteoporos. Rep. 7, 84-90.

Gennari, L., Merlotti, D., Valenti, R., Ceccarelli, E., Ruvio, M., Pietrini, M. G., et al. (2012). Circulating Sclerostin levels and bone turnover in type 1 and type 2 diabetes. J. Clin. Endocrinol. Metab. 97, 1737-1744.

Gerdhem, P., Isaksson, A., Akesson, K., and Obrant, K. J. (2005). Increased bone density and decreased bone turnover, but no evident alteration of fracture susceptibility in elderly women with diabetes mellitus. Osteoporos. Int. 16, 1506-1512.

Gregorio, F., Cristallini, S., Santeusanio, F., Filipponi, P., and Fumelli, P. (1994). Osteopenia associated with non-insulin-dependent diabetes mellitus: what are the causes? Diabetes Res. Clin. Pract. 23, 43-54.

Hamada, Y., Fujii, H., and Fukagawa, M. (2009). Role of oxidative stress in diabetic bone disorder. Bone 45(Suppl. 1), S35-S38.

Hamed, E. A., Faddan, N. H., Elhafeez, H. A., and Sayed, D. (2011). Parathormone-25(OH)-vitamin D axis and bone status in children and adolescents with type 1 diabetes mellitus. Pediatr. Diabetes 12 , 536-546.

Hamilton, E. J., Rakic, V., Davis, W. A., Chubb, S. A. P., Kamber, N., Prince, R. L., et al. (2012). A five-year prospective study of bone mineral density in men and women with diabetes: the Fremantle Diabetes Study. Acta Diabetol. 49, 153-158.

Hampson, G., Evans, C., Petitt, R. J., Evans, W. D., Woodhead, S. J., Peters, J. R., et al. (1998). Bone mineral density, collagen type 1 (alpha) 1 genotypes and bone turnover in premenopausal women with diabetes mellitus. Diabetologia 41, 1314-1320.

Herrero, S., Calvo, O. M., GarciaMoreno, C., Martin, E., San Roman, J. I., Martin, M., et al. (1998). Low bone density with normal bone turnover in ovariectomized and streptozotocin-induced diabetic rats. Calcif. Tissue Int. 62, 260-265.

Holloway, W. R., Collier, F. M., Aitken, C. J., Myers, D. E., Hodge, J. M., Malakellis, M., et al. (2002). Leptin inhibits osteoclast generation. J. Bone Miner. Res. 17, 200-209.

Horowitz, M. C., Xi, Y., Wilson, K., and Kacena, M. A. (2001). Control of osteoclastogenesis and bone resorption by members of the TNF family of receptors and ligands. Cytokine Growth Factor Rev. 12, 9-18.

Hwang, Y. C., Jeong, I. K., Ahn, K. J., and Chung, H. Y. (2012). Circulating osteocalcin level is associated with improved glucose tolerance, insulin secretion and sensitivity independent of the plasma adiponectin level. Osteoporos. Int. 23, 1337-1342.

Inaba, M., Nishizawa, Y., Mita, K., Kumeda, Y., Emoto, M., Kawagishi, T., et al. (1999). Poor glycemic control impairs the response of biochemical parameters of bone formation and resorption to exogenous 1,25-dihydroxyvitamin D3 in patients with type 2 diabetes. Osteoporos. Int. 9, 525-531.

Johansson, A. G., Lindh, E., Blum, W. F., Kollerup, G., Sorensen, O. H., and Ljunghall, S. (1996). Effects of growth hormone and insulinlike growth factor I in men with idiopathic osteoporosis. J. Clin. Endocrinol. Metab. 81, 44-48.

Kanazawa, I., Yamaguchi, T., and Sugimoto, T. (2010). Baseline serum total adiponectin level is positively associated with changes in bone mineral density after 1 -year treatment of type 2 diabetes mellitus. Metab. Clin. Exp. 59, 1252-1256.

Kanazawa, I., Yamaguchi, T., and Sugimoto, T. (2011a). Relationship between bone biochemical markers versus glucose/lipid metabolism and atherosclerosis; a longitudinal study in type 2 diabetes mellitus. Diabetes Res. Clin. Pract. 92, 393-399.

Kanazawa, I., Yamaguchi, T., Yamauchi, M., Yamamoto, M., Kurioka, S., Yano, S., et al. (2011b). Serum undercarboxylated osteocalcin was inversely associated with plasma glucose level and fat mass in type 2 diabetes mellitus. Osteoporos. Int. 22, 187-194.

Kanazawa, I., Yamaguchi, T., Yamamoto, M., Yamauchi, M., Yano, S., and Sugimoto, T. (2009a). Serum osteocalcin/bone-specific alkaline phosphatase ratio is a predictor for the presence of vertebral fractures in men with type 2 diabetes. Calcif. Tissue Int. 85, 228-234.

Kanazawa, I., Yamaguchi, T., Yamauchi, M., Yamamoto, M., Kurioka, S., Yano, S., et al. (2009b). Adiponectin is associated with changes in bone markers during glycemic control in type 2 diabetes mellitus. J. Clin. Endocrinol. Metab. 94, 3031-3037.

Kanazawa, I., Yamaguchi, T., Yano, S. Yamauchi, M., Yamamoto, M., and Sugimoto, T. (2007). Adiponectin and AMP kinase activator stimulate proliferation, differentiation, and mineralization of osteoblastic MC3T3-E1 cells. BMC Cell Biol. 8:51. doi:10.1186/1471-2121-8-51

Lacativa, P. G., and Farias, M. L. (2010). Osteoporosis and inflammation. Arq. Bras. Endocrinol. Metabol. 54, 123-132.

Lappin, D. F., Eapen, B., Robertson, D. Young, J., and Hodge, P. J. (2009). Markers of bone destruction and formation and periodontitis in type 1 diabetes mellitus. J. Clin. Periodontol. 36, 634-641.

Lee, N. K., Sowa, H., Hinoi, E., Ferron, M., Ahn, J. D., Confavreux, C., et al. (2007). Endocrine regulation of energy metabolism by the skeleton. Cell 130, 456-469.

Leite Duarte, M. E., and da Silva, R. D. (1996). Histomorphometric analysis of the bone tissue in patients with non-insulin-dependent diabetes (DMNID). Rev. Hosp. Clin Fac. Med. Sao Paulo 51, 7-11.

Leon, M., Larrodera, L., Lledo, G., and Hawkins, F. (1989). Study of bone loss in diabetes mellitus type 1. Diabetes Res. Clin. Pract. 6, 237-242.

Levy, L., Stern, Z., and Gutman, A. (1986). Plasma calcium and phosphate levels in an adult noninsulindependent diabetic population. $\mathrm{Cal}$ cif. Tissue Int. 39, 316-318.

Luo, X. H., Guo, L. J., Xie, H., Yuan, L. Q., Wu, X. P., Zhou, H. D., et al. (2006). Adiponectin stimulates RANKL and inhibits OPG expression in human osteoblasts through the MAPK signaling pathway. J. Bone Miner. Res. 21, 1648-1656.

Luo, X. H., Guo, L. J., Yuan, L. Q., Xie, H., Zhou, H. D., Wu, X. P., et al. (2005). Adiponectin stimulates human osteoblasts proliferation and differentiation via the MAPK signaling pathway. Exp. Cell Res. 309, 99-109.

Manolagas, S. C., and Almeida, M. (2007). Gone with the Wnts: beta-catenin, T-cell factor, forkhead box $\mathrm{O}$, and oxidative stress in age-dependent diseases of bone, lipid, and glucose metabolism. Mol. Endocrinol. 21, 2605-2614.

Mastrandrea, L. D., Wactawski-Wende, J., Donahue, R. P., Hovey, K. M., Clark, A., and Quattrin, T. (2008). Young women with type 1 diabetes have lower bone mineral density that persists over time. Diabetes Care 31, 1729-1735.

McNair, P., Madsbad, S., Christensen, M. S., Christiansen, C., Faber, O. K., Binder, C., et al. (1979). Bone mineral loss in insulin-treated diabetes mellitus: studies on pathogenesis. Acta Endocrinol. 90, 463-472.

Miazgowski, T., and Czekalski, S. (1998). A 2-year follow-up study on bone mineral density and markers of bone turnover in patients with longstanding insulin-dependent diabetes mellitus. Osteoporos. Int. 8, 399-403.

Miazgowski, T., Noworyta-Zietara, M., Safranow, K., Ziemak, J., and Widecka, K. (2012). Serum adiponectin, bone mineral density and bone turnover markers in post-menopausal women with newly diagnosed Type2 diabetes: a 12-month follow-up. Diabet. Med. 29, 62-69.

Motyl, K. J., McCabe, L. R., and Schwartz, A.V. (2010). Bone and glucose metabolism: a two-way street. Arch. Biochem. Biophys. 503, 2-10.

Munoz-Torres, M., Jodar, E., EscobarJimenez, F., Lopez-Ibarra, P. J., and Luna, J. D. (1996). Bone mineral density measured by dual X-ray absorptiometry in Spanish patients with insulin-dependent diabetes mellitus. Calcif. Tissue Int. 58, 316-319.

Neumann, T., Samann, A., Lodes, S., Kastner, B., Franke, S., Kiehntopf, M., et al. (2011). Glycaemic control is positively associated with prevalent fractures but not with bone mineral density in patients with Type 1 diabetes. Diabet. Med. 28, 872-875.

$\mathrm{Ng}$, K. W. (2011). Regulation of glucose metabolism and the skeleton. Clin. Endocrinol. (Oxf) 75, 147-155.

Okuno, S., Ishimura, E., Tsuboniwa, N., Norimine, K., Yamakawa, K., Yamakawa, T., et al. (2013). Significant inverse relationship between serum undercarboxylated osteocalcin and glycemic control in maintenance hemodialysis patients. Osteoporos. Int. 24, 605-612.

Oz, S. G., Guven, G. S., Kilicarslan, A., Calik, N., Beyazit, Y., and Sozen, T. (2006). Evaluation of bone metabolism and bone mass in patients with type-2 diabetes mellitus. J. Natl. Med. Assoc. 98, 1598-1604. 
Pedrazzoni, M., Ciotti, G., Pioli, G., Girasole, G., Davoli, L., Palummeri, E., et al. (1989). Osteocalcin levels in diabetic subjects. Calcif. Tissue Int. $45,331-336$.

Pondel, M. (2000). Calcitonin and calcitonin receptors: bone and beyond. Int. J. Exp. Pathol. 81, 405-422.

Puri, T., Blake, G. M., Curran, K. M., Carr, H., Moore, A. E., Colgan, N., et al. (2012). Semiautomatic regionof-interest validation at the femur in (18)F-fluoride PET/CT. J. Nucl. Med. Technol. 40, 168-174.

Raskin, P., Stevenson, M. R., Barilla, D. E., and Pak, C. Y. (1978). The hypercalciuria of diabetes mellitus: its amelioration with insulin. Clin. Endocrinol. (Oxf) 9, 329-335.

Rasul, S., Ilhan, A., Reiter, M. H., Todoric, J., Farhan, S., Esterbauer, H., et al. (2012a). Levels of fetuin-A relate to the levels of bone turnover biomarkers in male and female patients with type 2 diabetes. Clin. Endocrinol. (Oxf) 76, 499-505.

Rasul, S., Ilhan, A., Wagner, L., Luger, A., and Kautzky-Willer, A. (2012b). Diabetic polyneuropathy relates to bone metabolism and markers of bone turnover in elderly patients with type 2 diabetes: greater effects in male patients. Gend. Med. 9, 187-196.

Reid, I. R. (2002). Relationships among body mass, its components, and bone. Bone 31, 547-555.

Reid, I. R., Evans, M. C., Cooper, G. J., Ames, R. W., and Stapleton, J. (1993). Circulating insulin levels are related to bone density in normal postmenopausal women. Am. J. Physiol. 265, E655-E659.

Reyes-Garcia, R., Rozas-Moreno, P., Lopez-Gallardo, G., Garcia-Martin, A., Varsavsky, M., Aviles-Perez, M. D., et al. (2013). Serum levels of bone resorption markers are decreased in patients with type 2 diabetes. Acta Diabetol. 50, 47-52.

Rosato, M. T., Schneider, S. H., and Shapses, S. A. (1998). Bone turnover and insulin-like growth factor I levels increase after improved glycemic control in noninsulin-dependent diabetes mellitus. Calcif. Tissue Int. 63, 107-111.

Shu, A., Yin, M. T., Stein, E., Cremers, S., Dworakowski, E., Ives, R., et al. (2012). Bone structure and turnover in type 2 diabetes mellitus. Osteoporos. Int. 23, 635-641.

Suzuki, K., Ishida, H., Takeshita, N., Taguchi, Y., Sugimoto, C., Nosaka, K., et al. (1998). Circulating levels of tartrate-resistant acid phosphatase in rat models of noninsulin-dependent diabetes mellitus. J. Diabetes Complicat. 12, 176-180.

Tamura, T., Yoneda, M., Yamane, K., Nakanishi, S., Nakashima, R., Okubo, M., et al. (2007). Serum leptin and adiponectin are positively associated with bone mineral density at the distal radius in patients with type 2 diabetes mellitus. Metab. Clin. Exp. 56, 623-628.

Valerio, G., del Puente, A., Esposito-del Puente, A., Buono, P., Mozzillo, E., and Franzese, A. (2002). The lumbar bone mineral density is affected by long-term poor metabolic control in adolescents with type 1 diabetes mellitus. Horm. Res. 58, 266-272.

Verhaeghe, J., Suiker, A. M., Visser, W. J., Van Herck, E., Van Bree, R., and Bouillon, R. (1992). The effects of systemic insulin, insulinlike growth factor-I and growth hormone on bone growth and turnover in spontaneously diabetic BB rats. $J$. Endocrinol. 134, 485-492.

Vestergaard, P. (2007). Discrepancies in bone mineral density and fracture risk in patients with type 1 and type 2 diabetes - a meta-analysis. Osteoporos. Int. 18, 427-444.

Vestergaard, P., Rejnmark, L., and Mosekilde, L. (2009). Diabetes and its complications and their relationship with risk of fractures in type 1 and 2 diabetes. Calcif. Tissue Int. 84, 45-55.

WHO. (2012). Country and Regional Data. Available at: http://www.who.int/diabetes/ facts/world_figures/en/print.html $09 / 17$

Wikipedia. (2012). Diabetes Mellitus. Available at: http://en.wikipedia. org/wiki/Diabetes_mellitus 09/17

Wittrant, Y., Gorin, Y., Woodruff, K., Horn, D., Abboud, H. E. Mohan, S., et al. (2008). High $\mathrm{d}(+)$ glucose concentration inhibits RANKL-induced osteoclastogenesis. Bone 42, 1122-1130.

Yakar, S., Rosen, C. J., Beamer, W. G., Ackert-Bicknell, C. L., Wu, Y., Liu,
J. L., et al. (2002). Circulating levels of IGF-1 directly regulate bone growth and density. J. Clin. Invest. 110, 771-781

Zhou, Y., Li, Y., Zhang, D., Wang, J., and Yang, H. (2010). Prevalence and predictors of osteopenia and osteoporosis in postmenopausal Chinese women with type 2 diabetes. Diabetes Res. Clin. Pract. 90, 261-269.

Conflict of Interest Statement: The authors declare that the research was conducted in the absence of any commercial or financial relationships that could be construed as a potential conflict of interest.

Received: 05 November 2012; paper pending published: 04 December 2012; accepted: 22 February 2013; published online: 08 March 2013.

Citation: Starup-Linde J (2013)

Diabetes, biochemical markers of bone turnover, diabetes control, and bone. Front. Endocrinol. 4:21. doi: 10.3389/fendo.2013.00021

This article was submitted to Frontiers in Bone Research, a specialty of Frontiers in Endocrinology.

Copyright (c) 2013 Starup-Linde. This is an open-access article distributed under the terms of the Creative Commons Attribution License, which permits use, distribution and reproduction in other forums, provided the original authors and source are credited and subject to any copyright notices concerning any third-party graphics etc. 